\title{
Modeling Design and Application of Low-Temperature Plasma Treatment Test Stand for Seeds before Sowing
}

\author{
Changyong Shao ${ }^{1,2}$, Yong You ${ }^{1}$, Guanghui Wang ${ }^{1}$, Zhiqin Wang ${ }^{1}, \mathrm{Yan} \mathrm{Li}^{2}$, \\ Lijing Zhao ${ }^{2}$, Xin Tang ${ }^{3}$, Liangdong Liu ${ }^{1}$, and Decheng Wang ${ }^{1,4, *}$ \\ ${ }^{1}$ College of Engineering, China Agricultural University, Beijing 10083, P.R. China \\ ${ }^{2}$ Shandong Province Seeds Group, Jinan 250100, P.R. China \\ ${ }^{3}$ Shandong Agricultural and Engineering College, Jinan 250100, P.R. China \\ ${ }^{4}$ Key Lab of Soil-Machine-Plant System of Chinese Agriculture Ministry, Beijing 10083, \\ P.R. China \\ $\left\{\right.$ wdc, shaochangyong $\varrho^{\mathrm{a}} \mathrm{cau} . \mathrm{edu} . \mathrm{cn}$
}

\begin{abstract}
Stimulation with a low-temperature plasma (LTP) can improve the seed germination and seed adaptability to the environment. This technology has been applied in practice, but the study level of mechanism involved is still limited. Moreover, the treatment devices in using are quite simple. The paper focuses on the modeling design and application of the LTP treatment test stand for seeds before sowing. Numerous experimental LTP treatment on crop seeds and forage grass seeds will be conducted to find out the optimal dose used in the treatment. Another advanced method of this modeling is data collecting and intelligent monitoring system designed in, therefore all key process parameters can be under control during treatment period. At the same time, the modeling design will provide technical support for large-scale manufacturing of seed treatment devices.
\end{abstract}

Keywords: digital intelligence, Low-temperature Plasma(LTP), seeds treatment before sowing, development trend.

Studies show that the application of high quality, specially prepared seed is an important, yet still underestimated, yield-forming factor in the cultivation of many species of agricultural plants.Chemical methods are frequently applied in the treatment of seeds prior to sowing,but these methods are recognised as harmful to the environment because they are not selective in respect of nature lives although the agro-chemicals introduced are often very efficient. Nowadays, more and more physical methods (such as low-temperature plasma (LTP), magnetic technology, bio-frequency spectrum irradiation, solar power) have attributed to the pre-sowing treatment of seeds $[1,2,3,4,5]$ which are commonly regarded as being friendlier to the environment.

\footnotetext{
* Corresponding author.
} 


\section{Introduction}

The main objectives of this article are to prove the effect of low-temperature plasma treatment on seed germination and seedling, including vigor and rate of germination, root system, resistance to disease and drought,plant quality, maturity and yield; to design a modeling of low-temperature plasma treatment test stand for crop seeds and forage seeds. A scientific parameters of the technology system will be established for this stand,which will play a important role in the analysis and selection of treatment doze for different crop seeds, data collecting and intelligent monitoring system is the other major part of the test stand making all key parameters under supervision and control. Such kind of treatment devices can be produced in a large scale once the LTP test stand in the article is established and proved reliable.

Physical agriculture technology is the application of physical methods in agriculture and has been studied since the 1970s, many reachers wrote articles about this. Mr.Yin reported that low temperature plasma technology was utilized in biological applications in Russia, but there had been little research into the mechanisms involved [6]. According to Zhang, plasma seed treatment represented a new technology which could activate endogenous substances in the seeds, leading to improvements in the rate of seed germination, crop resistance, crop yield and an earlier maturity date [7]. According to $\mathrm{Ms}$.Xu and Mr.Chen, plasma processing could be applied to the principle of space breeding and seed treatment with plasma energy and alternating inductance would stimulate the seeds potential and improve their vigor and strength [8]. Mr. Liu hypothesized that this technology narrowed the differences between individual seeds [9]. Mr.Li believed that plasma energy could separate oxygen molecules in the air and form ozone. Bacteria attached to the surface of seeds would be disinfected under the action of both plasma and ozone [10]. Laboratory tests have confirmed that for low plasma energy and a limited treatment time, no variations occur in the treated seeds and no character changes are observed during the plant growth period of the treated seeds[11].

As the mechanism of low-temperature plasma effect on seeds is not very clear, and the LTP processing device is quite simple now (Figure 1), it becomes impossible to use this technology and devices in a large scale. However, this question also make low-temperature plasma treatment before seed sowing become into a very attractive project to explore, a large number of lab tests have been done in lab and small open field by the authors, all results prove that plants show better characteristics in terms of germination, root system, resistance to drought and diseases, early maturity and yield after their seeds well treated by a suitable dose of low-temperature plasma (LTP). 


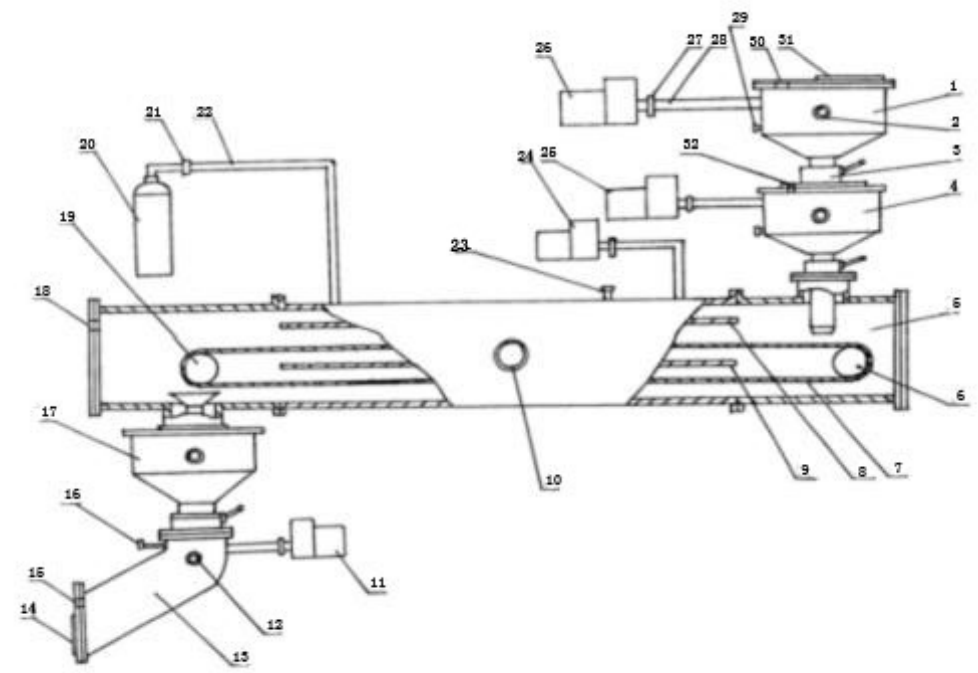

Fig. 1. Diagram of low-temperature plasma test stand device

1, Feeder compartment I; 2, Detector tube I; 3, Butterfly gate; 4, Feeder compartment II; 5, Feeder compartment III; 6, Driven wheel; 7, Transmission belt; 8, Top crown; 9, Bottom crown; 10, Detector tubeII; 11, Vacuum air pump I; 12, Detector tube III; 13, Feeder compartment IV; 14, Discharge port; 15, Thermocouple gauge tube I; 16, Deflating valve I; 17, Feeder compartment V; 18, Thermocouple gauge tube II; 19、Driver wheel; 20, Gas tank; 21, Gate; 22, Inlet pipe; 23, Deflating valve II; 24, Vacuum air pump II; 25, Vacuum air pump III; 26, Vacuum air pump IV; 27, Electromagnetic relief valve; 28, Vacuum tube; 29, Deflating valve III; 30, Thermocouple gauge tube III; 31, Feed cap; 32, Thermocouple gauge tube IV.

\section{Key Points Focused in the Article}

Shao Changyong (2012) pointed out that digital intelligent would be the development trend of the plasma seed processing device. To meet with this trend, the article focuses on two parts as following:

Firstly, the effects of different dose of LTP treatment on different crop seeds should be studied to find out the most appropriate dose applied in treatment. Secondly, increase stability of the LTP platform and carry out that data acquirement and signal analysis with computer, perfect the LTP system, achieve safety and reliable operation. 


\section{Contents and Objectives Decomposed}

\subsection{Contents of the Research}

(1) General design. To study and summarize all scientific reports and articles concerned worldwide, clarify the basic function of LTP treatment devices for seeds and define the designs for both test stand and Experiment involved.

(2) Study on the design of LTP test device. This design composed by two parts:design of hardware and design of software. Hardware part will be responsible for technical treatment of LTP, while software part will charge of data collecting, supervising, controlling in the treatment period, and provide technical support for the intelligent devices in the future.

(3) Find out the appropriate dose of LTP. 30 different crop seeds and forage seed with low germination will be treated by different doze of LTP in our study, germination rate of treated and untreated seeds will be compared, finally to find out the relationship between characters of seeds and the treatment doze of LTP

(4) Communication between soft system and PC Visual Basic language will be used in software system design, and the communication between software system and PC will be realized through serial circuit.

\subsection{Research Goal}

(1) To submit more study results in the LTP research field. Take certain corp seeds and forage seed as the research targets whose biological germinations are poor, the article believes that find out the most ideal treatment dose can solve the technical barrier of promoting LTP treatment devices into the agricultural application.

(2) To provide craft and technical parameters for the research of technology integration. Integration of seed processing, mechanics engineering, computers and informations is the development trend of agricultural technology, the article is trying to establish such a reliable, intelligent model of LTP treatment device on the ground of massive experiments and to provide craft and technical parameters for the upgrading of seed treatment devices.

\section{Research Plan}

\subsection{Research Methods}

As many discipline involved in the project, six methods will be used in the research:

(1) Method of scientific literature review and survey analysis

(2) Method of survey analysis and experiment

(3) Method of experimental and positive research

(4) Method of agronomy research and agricultural engineering research

(5) Method of hardware design and software design

(6) Method of general design and each module design 


\subsection{Technical Route}

The route of modeling design and application of the LTP treatment test stand is shown in figure 2 .

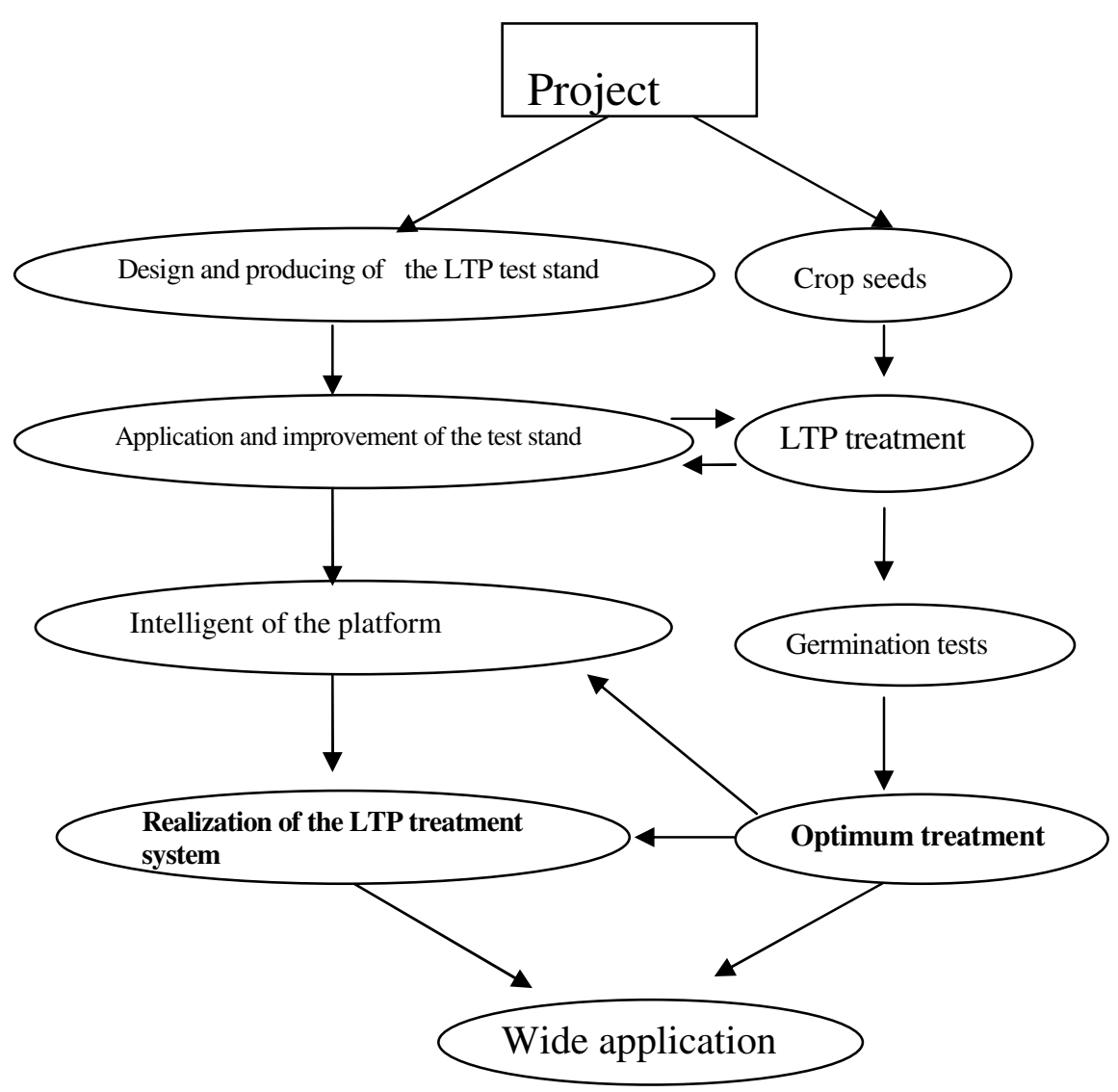

Fig. 2. LTP treatment system design

\subsection{Scheme of the Experiments}

\subsubsection{Sample Seeds for the Experiments}

All sample seeds are chosen from crop seeds and forage grass seeds, whose natural germinations are poor but uniform in size with good viability.

\subsubsection{Treatment Dose of LTP}

Healthy and uniform seeds will be counted and treated with various LTP strength $(0,10,20,40,60,80,100,120,140,160,200,220,240,260,280,300,320,340,360$, $380,400 \mathrm{~W})$. 


\subsubsection{Germination Test}

Germination test for vegetable seeds and small forage grass seeds: treated seeds will be placed in petri dish with two-layer filter paper at the bottom, 100seeds in each petri dish, and $4 \mathrm{ml}$ steamed water added in the dish to keep fine moisture. Germination test of the treated seeds will be done in the incubator with suitable temperature along with a check test. Each test will be repeated four times to confirm the repeatability of the results.

Germination test for grain seeds and large-size forage grass seeds: 50 treated seeds will be placed in petri dish with $120 \mathrm{gr}$ sterile sands at the bottom and $30 \mathrm{gr}$ sands on the top of the seeds for coverage, $30 \mathrm{ml}$ water will be dripped in, petri dish will be put on a light culture shelf, light period will be 12 hours. The lid of petri dish should be uncovered after 3 days, $10 \mathrm{ml}$ water is designed to drip in every 2 hour to keep sufficient water supply. Each test will be repeated four times and a check test is also added in.

Determination method of the germination

The germination rate is defined as the percentage of seeds that germinate in a specified short time (ST), the time in the test is specified as the days from the day of the first cotyledons breaking out from the seed coats or covered sands to the day that no new cotyledons coming out in 3days. These are calculated as follows:

Vigor of germination $(\%)=($ Number of seeds germinated in ST days/total number of seeds) $\times 100 \%$.

Rate of germination $(\%)=($ Number of seeds germinated $/$ total number of seeds $) \times$ $100 \%$.

\subsubsection{Determination of the Optimal Dose of LTP Treatment for Various Species Seeds}

After germination test, the data of optimal intensity of LTP treatment for various species seeds will be got, list them in the following table:

Table 1. Optimal intensity of LTP treatment for seeds

\begin{tabular}{lllllllll}
\hline $\begin{array}{l}\text { Crop varieties } \\
\text { Intensity of }\end{array}$ & V1 & V2 & V3 & V4 & V5 & V6 & V7 & V8 \\
$\begin{array}{l}\text { LTP treatment } \\
(W)\end{array}$ & W1 & W2 & W3 & W4 & W5 & W6 & W7 & W8 \\
\hline
\end{tabular}

\subsubsection{Software Design and Communication with PC}

Modularization method for designing software program will be based on the determination of optimal doses of LTP treatment for different seeds and construction of LTP treatment technology system, and it is in favour of extending and debugging a new function (Figure 3). The system and communications software will be programmed with the Windows XP operating system and for most stand-alone PC users, the interface is friendly and easy to operate. 


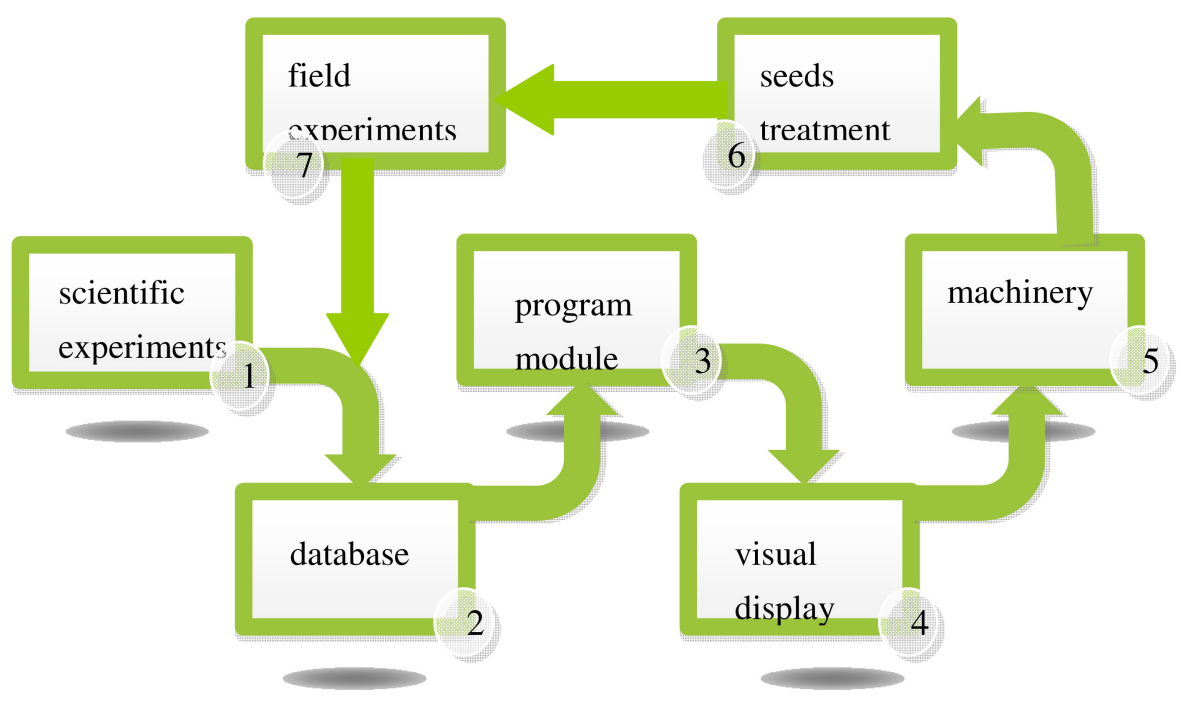

Fig. 3. Software programme design flowchart

\section{Conclusions}

(1) The paper try to establish a modeling of LTP treatment test stand for seeds before sowing, aiming at realizing a intelligent control of treatment dose, processing operation, supervision, data collecting and information feedback during the whole treatment.

(2) The paper try to determinate the optimal doses of LHP treatment and other key technical parameters for LTP treatment, finally find out the technical access to the research and development of LTP treatment machinery.

LTP treatment, as one of the effective physical methods for preparing seed, has an significant meaning to stimulate the potential energy of seeds, an intelligent LTP treatment device will meet this requirement. As an technologies integration of computer, machinery and serial communication, the new improved LTP treatment researched in this paper will be more stable and effective. It will be the development trend in agriculture industry and has a widely applied prospects.

Acknowledgment. The study was supported by Special Fund for Agro-scientific Research in the Public Interest (201203024) and the Fundamental Research Funds for the Central Universities(NO.2013QJ019).

\section{References}

1. Phirke, P.S., Kudbe, A.B., Umbarkar, S.P.: The influence of magnetic field on plant growth. Seed Science and Technology 24(2), 375-392 (1996)

2. Pietruszewski, S.: Effect of magnetic seed treatment on yield of wheat. Seed Science and Technology 21(3), 621-626 (1993) 
3. Podleoeny, J.: The effect pre-sowing treatment of laser light on morphological features formation and white lupine yielding. In: Lupin, An Ancient Crop for the New Millenium. Department of Agronomy and Soils, Alabama Agric. Expt. Stn. and Auburn University, pp. 388-390 (1999)

4. Shao, C., Wang, D., Tang, X., et al.: Stimulating effects of magnetized arc plasma of different intensities on the germination of old spinach seeds. Mathematical and Computer Modelling 58(3-4), 808-812 (2013)

5. Shao, C., Fang, X., Tang, X., et al.: Effects of low-temperature plasma on seed germination characteristics of green onion. Transactions of the Chinese Society for Agricultural Machinery 44(6), 212-216 (2013) (in Chinese with an English abstract)

6. Yin, M.: Research of magnetized arc plasma on seeds biological effects. Dalian University of Technology, Dalian (2006) (in Chinese)

7. Zhang, Y., Zhang, J., Wang, Q.: The application of physical methods in sugar beet seed treatment. China Beet and Sugar (2), 20-22 (2005) (in Chinese )

8. Xu, Z., Chen, B., Wei, Z.: Various seed treatments on corn yield. Agricultural science \& Technology and Equipment (4), 15-16 (2011) (in Chinese)

9. Liu, S., Ouyang, X., Nie, R.: Application status and development trend of the physical methods in the crop seed treatment. Crop Research 5(2), 520-524 (2007) (in Chinese)

10. Li, R.: Plasma machine seed treatment technology. North Rice 4(4), 52-53 (2010) (in Chinese)

11. Fu, S., Zhang, F., Li, J., et al.: Several physical techniques in agriculture and Prospects. Agricultural Mechanization Research (1), 36-38 (2006) (in Chinese) 